\title{
On the impact of lightweight ciphers in automotive networks
}

\author{
Arcangelo Castiglione*, Francesco Palmieri ${ }^{\dagger}$ \\ Department of Computer Science \\ University of Salerno \\ arcastiglione@unisa.it ${ }^{*}$,fpalmieri@unisa.it
}

\author{
Francesco Colace ${ }^{\S} \|$, Marco Lombardi ף, \\ Domenico Santaniello** \\ Department of Industrial Engineering \\ University of Salerno \\ fcolace@unisa.it ${ }^{\natural}$, malombardi@unisa.it $^{\natural}$, \\ dsantaniello@unisa.it ${ }^{k *}$
}

\begin{abstract}
The ever more exposure of modern vehicles to computer networks has led, in recent years, to increase risks due to cyberattacks. The internal computer network of vehicles, used to connect several electronic components present on cars, is even more threatened as it is potentially exposed to external attacks. Securing the Controller Area Network (CAN) protocol, used to govern those networks, is becoming increasingly important to ensure a safe driving experience.

CAN is an ISO standard that dates back to 1983, over the years it has undergone very few changes coming to be outdated. It has been designed to minimize latency and data transmission errors through two essential features: small frames and unencrypted information transfer. The latter feature, in particular, appears to be the weak point of this protocol. Securing the communication channel is needed, but it must be done by preserving all the main features that ensure the performance of this protocol, in particular the low latency. Furthermore, the modification cannot introduce low-level alterations.

CAN security can be improved by acting at a higher level. In this work we investigate the feasibility of using symmetric encryption algorithms for securing messages exchanged on the CAN-bus. In particular, this paper evaluates the effectiveness of using lightweight ciphers, designed with the aim of introducing encryption also on devices, which have limited hardware and software resources, such as microcontrollers.
\end{abstract}

Keywords-Automotive security; Controller Area Network; Lightweight Cryptography; Cybersecurity; Encryption.

\section{INTRODUCTION}

We live in the Internet of Things (IoT) era, in which innumerable devices are interconnected and connected to the Internet, constantly exchanging information for several purposes [1]-[6]. In particular, IoT indicates a set of technologies that allow any type of device to be connected to the Internet and interact. The purpose of this type of solutions is varied [7]-[13] and could concerns monitoring, controlling and transferring information to perform consequent actions. The automotive field, not least, has progressively invested in the IoT paradigm. In fact, modern vehicles are equipped with many sensors that enhance their features and services. It is estimated that by 2022 new vehicles will be able to communicate with each other [14]. Modern vehicles are able to exchange information over the network using wireless technologies and cloud services. However, these communication channels expose the vehicle to

\footnotetext{
II Corresponding author: Francesco Colace, Department of Industrial Engineering, University of Salerno, fcolace@unisa.it Via Giovanni Paolo II, 132 I-84084 Fisciano (SA), ITALY. Phone: +39089964256, Fax: +39089964218
}

potential vulnerabilities that may prejudice their functionality by attacking the internal network [15], [16].

Internet access exposes vehicles to potential attacks that can be different and varied. An attacker may be interested in spying on users to find personal information or even remotely control theirs vehicle. The Controller Area Network (CAN) represents the internal network of the vehicle which, in modern vehicles, consists of 70 nodes, which are the Electric Control Units (ECUs). The ECUs are responsible for controlling each component of the vehicle, from the ventilation system up to the braking system and steering [17], [18]. The communication channel, called CAN-bus, collects the exchange broadcast information between the various ECUs. Therefore, access to the CAN-bus would compromise the entire safety of the vehicle [19], [20].

The CAN-bus security issue has been addressed in the literature in several ways. One of the approaches is to redesign the CAN standard from a hardware perspective. However, this type of solution, with the exception of a substantial hardware upgrade, brings the disadvantage of not ensuring security to all vehicles already on the market [21]. Different approaches aim to equip the internal vehicle network with an Intrusion Detection System (IDS), which however, in many cases exceed the computational capacity of the microcontrollers [22], [23]. In attempt to preserve the exchange of information unchanged and considering the insufficient computational capability of microcontrollers, also possible solutions based on the Message Authentication Code (MAC) are to be excluded [24].

The aim of this work is to envision a solution for securing messages exchanged on the CAN-bus throught symmetric encryption algorithms. In particular, a solution using lightweight ciphers is proposed and evaluated. Lightweight ciphers provide encryption facilities on devices which have limited hardware and software resources, such as microcontrollers.

With the aim of extending our solution to all vehicles already on the market, the selected lightweight ciphers are PRESENT [25], SIMON and SPECK [26], which should produce safety improvement with little delay in communications. With the goal of evaluating the proposed approach, a prototype using Automotive Grade Linux (AGL) [27] was developed to evaluate the impact of the proposed solution on system performance. This paper is organized as follows. In Section $\Pi$ we provide the background underlying the proposed approach. In Section III we provide our solutions in order to protect modern vehicles. In Section IV we describe a proof of concept of our proposed solution. In Section $\mathrm{V}$ we discuss a preliminary test activity. 
Finally, in Section VI we draw conclusions and future research directions.

\section{BACKGROUND}

\section{A. The Controller Area Network Standard}

The Controller Area Network bus, also known as CAN-bus, is a robust bus standard used to allow the interconnection of Electronic Control Units (ECUs), which controls all electronic vehicle systems [28].

The CAN-bus is used manly in automotive sector, although it is adopted also in other applications, such as industrial automation or healthcare industry.

The CAN protocol was designed around the 1980s at the Bosh laboratories. In the following years, Bosh released a new version of the protocol that was published as the ISO 11898 standard [29]. The protocol was designed to interconnect ECUs components, one by one in an economic and efficient way. The CAN is based on a broadcast network through which all the ECUs listen and communicate the new messages. In this way, the CAN protocol preserves the efficency needed in real-time applications such as automotive sector.

1) CAN Architecture: $\mathrm{CAN}$ is designed to minimize the number of messages reducing overhead and lost messages amount, thus ensuring as efficient as possible communication. The protocol provides a hierarchy among the messages exchanged on the channel, through priorities avoiding, of critical messages during simultaneous transmission of other less important signals. Frames (also called messages) perform the actual data transmission. There are four types of frames that can be transmitted on the CAN-bus: data, remote, error and overload.

(a)

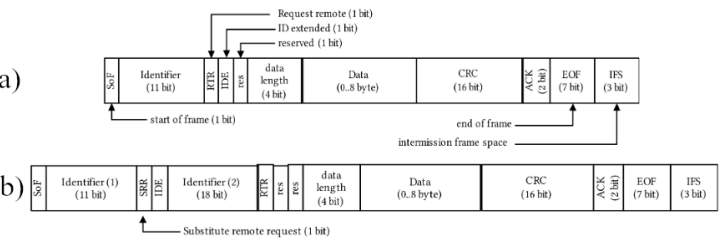

Fig. 1: Data Frames CAN 2.0: (a) Standard, (b) Extended.

The messages can be in two formats: basic frame format (shown in Fig. 11) with 11 bits and extended frame format (shown in Fig. 15) with 29 identification bits. The data frame is responsible for transmitting data and no more than 64 bits of information can be transferred without encryption.

In the design phase, the strong presence of electromagnetic interference in the operating environment and the presence of priority relationships between the messages were not neglected. In fact, there is a very low probability that the messages exchanged on the CAN-bus suffer alterations due to the city electromagnetic fields. It is also possible to define priorities, so as to prevent critical signals from being lost due to the simultaneous transmission of other less important messages. The interconnection is made physically through the use of a twinned pair to which all nodes involved are connected. The latter consists of a CPU, capable of decoding incoming messages and encoding those in output, by a CAN controller, which deals with the recomposition of the input message and the serialization of the output bits towards the CAN-bus (often this module is integrated in the CPU), and by a transceiver, which carries out a translation of the data stream in a format understandable by the CAN controller.

\section{B. Automotive Grade Linux}

The variety of software and hardware of the vehicles on the market is vast. Each manufacturer tends to propose different solutions both for mechanical and electronic system, which govern the vehicles. This generates fragmentation by not allowing the use of unique software. Automotive Grade Linux arises against the software segmentation present in the automotive sector, offering an open source solution based on Linux [30]. AGL promises to improve safety and entertainment of vehicles through APIs present at all levels. The software started in 2012 by the Linux Foundation with the support of distinguished companies of automotive sector. Today AGL counts on more than hundred participating companies. Toyota was the first, in 2018, to produce Carmy, the first vehicle with AGL on board, which represents one of very successful sedans in the world.

1) Message Exchange: Automotive Grade Linux (AGL) offers an API service, which allows managing the CAN-bus interaction as shown in Fig. 2. This service is divided as follow: CAN High Level Binding(s), which deals with the applications user layer that offers logic and advanced functions; CAN Low Level Binding(s), which interacts with low-level communication, such as encoding and decoding of messages, security checks, transactions, etc. AGL, which presents an architecture

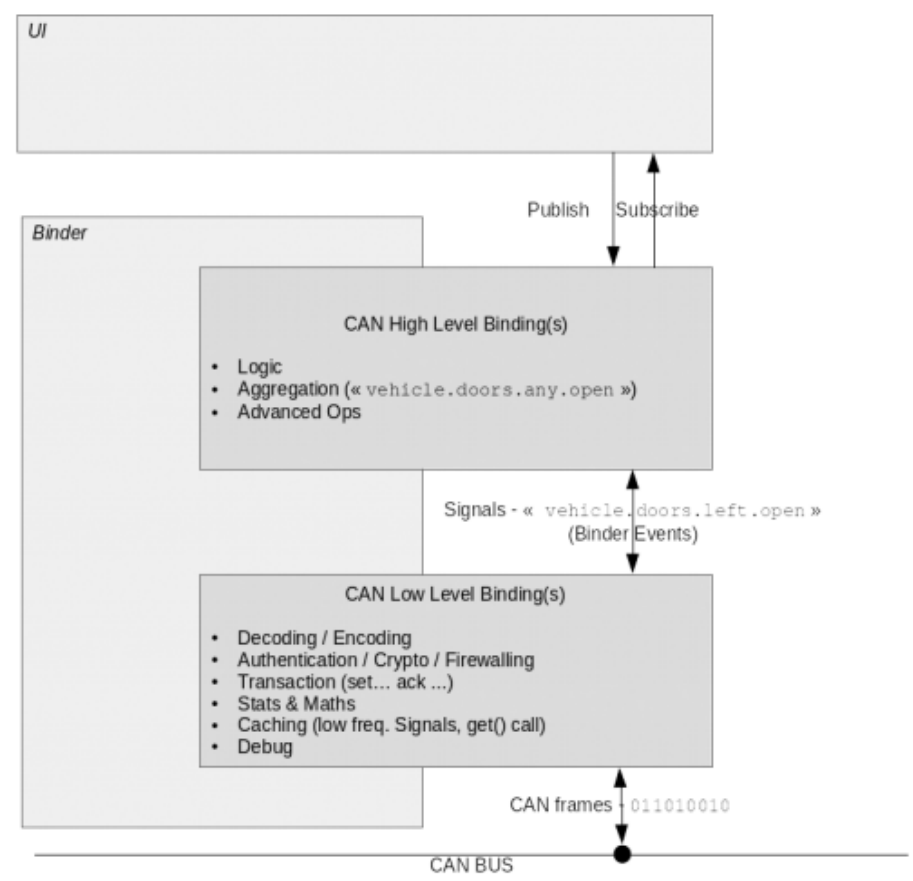

Fig. 2: Exchanging Message Architecture [27].

similar to the one defined in the OpenXC project [31] designed by Ford, offers convenient management of interactions with the CAN bus. The API for the exchange of messages is divided 
into two levels, that is high and low. The former deals with the logic and advanced functions that are offered to user applications, whereas the latter carries out security checks, transactions, caching, encoding and decoding of messages. The system is in fact designed to extract the various messages that can be admitted starting from a JSON file. Therefore, the applications require the transmission of a signal simply by using the name assigned to them during compilation. The lowest level of the CAN API then deals with the translation of this string into a frame of data and vice versa. The services are defined in files that are used by the afb-daemon binder and the latter can be queried via HTTP or WS specifying the name of the API to be used followed by the desired method. The actual writing and reading on/from the communication channel is done using the native Linux drivers.

\section{Proposed Solution}

This section deals with the proposed solution, which aims to protect connected vehicles from possible attacks. In fact, an attacker, through the use of malware, could attacks and controls an entire fleets of vehicles. Furthermore, it is necessary pay careful to make changes that do not compromise the performance of the protocol. In other words, security must be increased without compromising the efficient execution of message exchange. [32]

Four types of messages can be exchanged on the CAN bus, but the only one on which encryption could be applied is the data frame, which is involved in the exchange of information. However, the length of the data field is often of variable length and does not exploit all transmissible bytes. In this regard, to improve the resistance of the system to brute force attacks, it is necessary to use all the 64 bits available, using dummy data in order to introduce noise.

However, in the use of cryptographic algorithms, which would improve vehicle safety, there are very important limitations. Microcontrollers, i.e., hardware with limited memory capacity, the need to preserve the performance of the CAN protocol and the limited amount of data to be encrypted are the main limitations to consider when choosing ciphers. Lightweight ciphers are the best solution in the conditions described above. The main feature of lightweight encryption is to find the best compromise between security and lightness, reducing the resources needed to execute the algorithm, both in terms of space and time. In our case, due to the type of application, it is necessary to use ciphers capable of working on low performance hardware and with a particularly high encryption and decryption speed. These main features are peculiar to be implemented in the automotive sector. Some of the most wellknown lightweight ciphers are PRESENT [25], SIMON and SPECK [26].

\section{A. Protection of CAN Data Frames}

The field to consider, to prevent that an attacker takes control of the vehicle, is the data frames field, which contains information exchanged to all nodes on the internal network. Therefore, encrypting this field could be crucial for security improvements. We remark that for performance reasons, the protocol tends to transmit the minimum number of bits needed for each message. In fact, most of the messages exchanged on the bus do not use all the 64 bits available. However, for reasons related to security and correct use of ciphers, it is necessary to include dummy data to fill the entire field. It must not be forgotten that this operation could compromise the performance of the CAN protocol.

The proposed solution includes the using of a fixed key [33], [34], usable for the entire life of the vehicle or to be changed at regular intervals, to encrypt the messages exchanged on the CAN-bus. In fact, this solution does not imply hardware changes or synchronization problems between the different components responsible for communication. This key must be generated during production, remaining secret for the entire life of the vehicle.

However, if an attacker could discover the key, the safety of the entire vehicle would be compromised [35]. In this regard, the use of a fixed key is not very recommendable and this key should be changed at regular intervals.

The proposed solution involves the addition of a small operation during the production phase of the vehicle. Using a fixed key implies a little change in the production chain between the vehicle manufacturer and electronic component suppliers. More precisely, the latter will have to provide a software for flashing the firmware of the individual ECU, allowing the inclusion of the key during the assembly phase. Finally, we point out that although the key must be kept secret, however, it must be available for technical interventions such as replacing an ECU.

\section{EXPERIMENTAL PHASE}

To implement the proposed solution it is necessary to modify the modules dedicated to writing and reading messages on the CAN-bus, present in Automotive Grade Linux. It was therefore necessary to modify these modules by inserting an encryption phase after writing and a decryption phase before the reading. AGL is an open source environment, so it allows us to clone the source of the modules by modifying them as necessary.

In AGL, the low CAN service module is responsible for communication with the CAN-bus, through which it is possible to read, write and subscribe signals via HTTP or WS interface. This module could be modified at various points, to make the safety improvements proposed. The appropriate solution concerns to intervene at the binding definition level, making changes to the functions responsible of sending and receiving a message, without changing the embedded libraries.

\section{A. Message Encryption}

The binding is defined in the file low-canbinding/binding/low-can-cb.cpp and is designed in such a way that the writing of messages is managed by two different functions, depending on the mode used. In particular, the function write_signal() is invoked when we specify only the name and value of the signal to be sent. The writing of a raw frame instead passes through the function write_raw_frame(). Both of the functions mentioned above invoke the function send_frame(), which represents an excellent point to enter and carry out the encryption before the frame is passed to the sending module [36].

\section{Listing 1: Encryption in writing phase}

static int send_frame (struct canfd_frame\& cfd, const std:: string\& bus_name) 


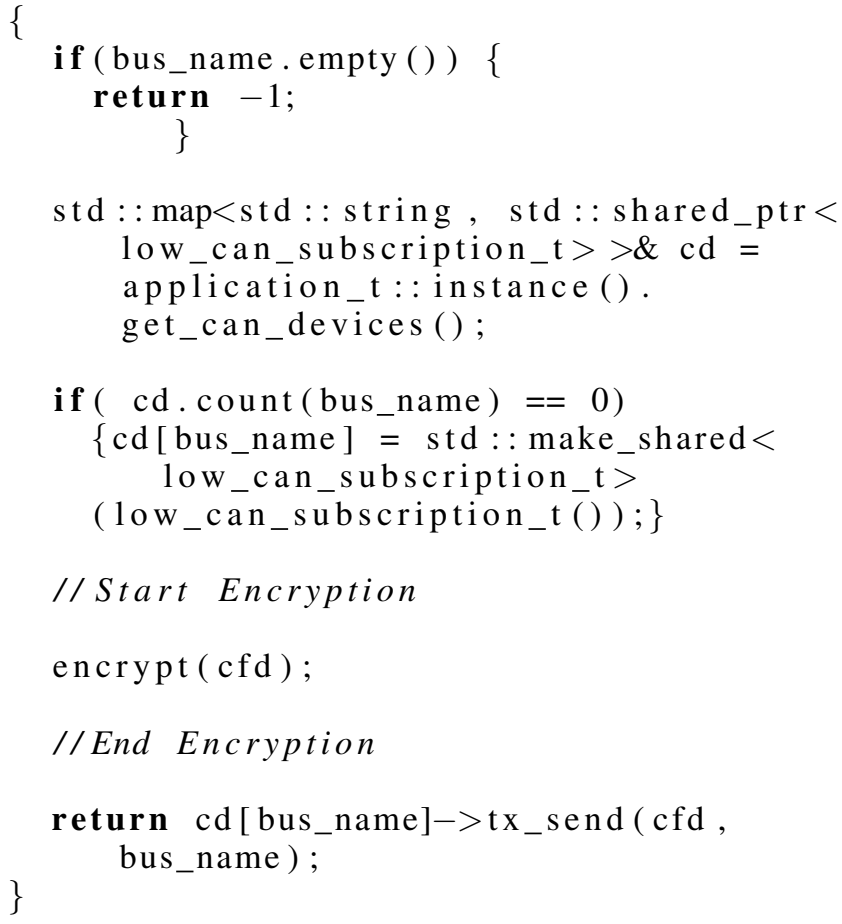

The modification consists simply in accomplishing, before the end of the function send_frame(), the encryption of the field data and eventually the modification of the value of the field len, keeping it consistent with the number of bytes actually used in data. In fact, the structure received as a parameter by the function send_frame() has several fields, among which there is data containing the raw information of the message and len which indicates the length of the message. In the code shown in Listing 1, the structure is passed as a parameter to the function encrypt() that inside it must provide for the implementation of the chosen cipher scheme and, if necessary, for the insertion of dummy data and for the modification of the len field.

\section{B. Message Decryption}

Intervention in reading requires a modification to the class can_message_t in order to make the vector editable data_. In fact, in this field the raw information is processed at a higher level. The modification concerns both the header in which the class is defined, and the file in which the behavior of the various methods is defined. The files in question are, respectively, low-can-binding/can/can-message.hpp and lowcan-binding/can/can-message.cpp [36].

Listing 2: Adding set in prototype in can-message.hpp void set_data_vector ( std : : vector $<$ uint 8 t $>\&)$

Listing 3: Implementation of the method in can-message.cpp void can_message_t : : set_data_vector ( std : : \{ vector $<$ uint $8_{-} t>\&$ data $)$
Once the problem of updating the data field has been solved, it is possible to intervene again in the definition of the binding, so as to define the decryption operation. In practice, the point in which to make changes is within the function read_message () .

Listing 4: Decryption in reading phase

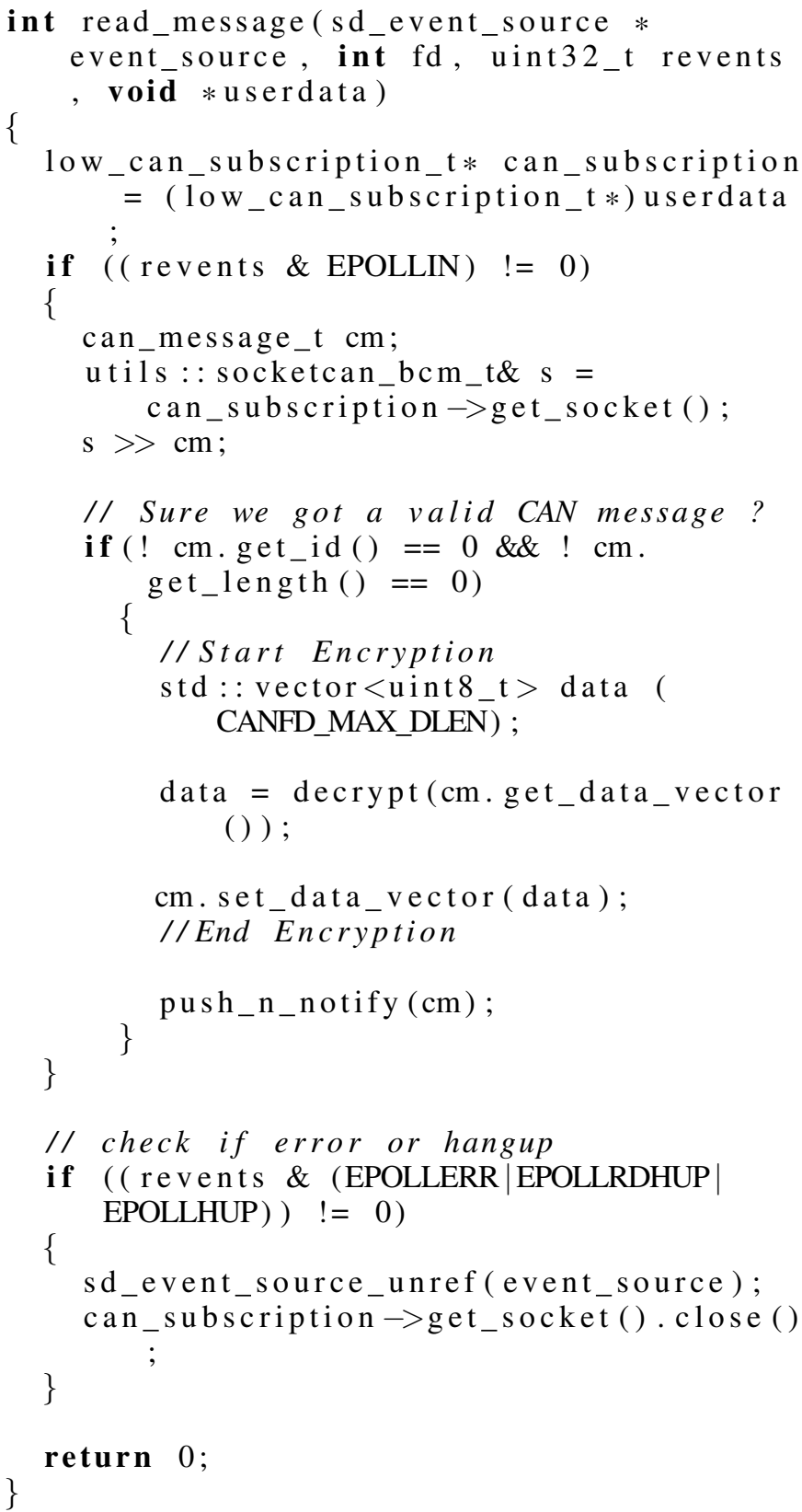

It remains to define the encryption mode, according to those chosen for the encryption process.

\section{EXPERIMENTAL RESULTS}

The function system_time_us() is used to obtain the delay between the reading and writing phases of a message exchanged on the CAN-bus. This function is used before the encryption and after decryption phases, providing the time spent during the entire process. The time obtained represents the delay brought by the use of the proposed methodology. 
The average of time obtained is between $300 \mu \mathrm{s}$ and $1 \mathrm{~ms}$ and, according to [37], it is more efficient to use hardwareoriented ciphers than the software one. To give an idea of the two effects to the delay generated by our solution, we can refer to a fundamental phase of driving a vehicle, which is the braking phase. The proposed solution generates delays in communication, which we can influence the various phases of driving the vehicle. Even if these delays are very low, to appreciate their significance we consider a crucial phase, which is the braking phase, transforming the delay in communication in terms of distance accumulated. However, even if, in the worst case, $1 \mathrm{~ms}$ represents a very low value, it causes an extra distance to travel of about $40 \mathrm{~mm}$, in terms of break reaction if a driver was travelling at $150 \mathrm{Km} / \mathrm{h}$ of speed. The delays obtained in terms of distance, in the best $(300 \mu \mathrm{s})$ and in the worst $(1 \mathrm{~ms})$ case, are reported in Table I.

TABLE I: Excess distance due to the delay introduced by the encryption

\begin{tabular}{|c|c|c|}
\hline $\begin{array}{c}\text { Speed } \\
{[\mathbf{K m} / \mathbf{h}]}\end{array}$ & $\begin{array}{c}\text { Distance (300 } \mu \mathbf{s}) \\
{[\mathbf{m m}]}\end{array}$ & $\begin{array}{c}\text { Distance (1ms) } \\
{[\mathbf{m m}]}\end{array}$ \\
\hline 50 & 4.2 & 13.9 \\
100 & 8.3 & 27.8 \\
150 & 12.5 & 41.7 \\
200 & 16.7 & 55.5 \\
250 & 20.8 & 69.4 \\
300 & 25.0 & 83.3 \\
\hline
\end{tabular}

According to the Table 1 , the results are encouraging. There are two main causes of the accumulated delay during the encryption and decryption phases, that are the algorithm used and the hardware on which the system is installed. The realized prototype has been installed on a Raspberry Pi 3 to simulate a generic ECU. However, on the vehicles present in today's market, microcontrollers are installed that could be even less efficient.

\section{CONCLUSIONS}

The study conducted in this paper deals with a cybersecurity problem in the automotive sector. In particular, was analysed the problem of the exchange of unencrypted messages on the CAN-bus and the feasibility of encryption that at least manages to prevent attacks on vehicles. According to the literature, an attacker who remotely penetrates into a vehicle's communication channel has the same control level of the driver. In this paper was analysed how important it can be to secure the CAN-bus, by obfuscating messages without altering their structure. This choice arises from the desire to keep the proposed solutions compatible with all the devices on the market, opening, in fact, to the possibility of correcting the security problem even on vehicles that already populate our roads. It has therefore been seen how message encryption is possible for all transmitted identifiers, as well as for sections containing data. We remark that due to the application field, the choice of the cipher is rather important, ensuring the least possible delay. Therefore, the use of lightweight ciphers has been evaluated. The study also focused on the implementation of a prototype through Automotive Grade Linux operating system and the interactions between the latter and the CANbus, achieving reasonable results. In fact, the maximum delay, introduced of our solution, is $1 \mathrm{~ms}$ and if the vehicle travels at $150 \mathrm{Km} / \mathrm{h}$ of speed, the extra distance generated by the delay is about $40 \mathrm{~mm}$, which is a reasonable value. Further considerations on error handling in the decryption phase are demanded to future steps.

\section{ACKNOWLEDGMENT}

The authors would like to thank Aniello Carotenuto, who developed a preliminary version of our proposal.

\section{REFERENCES}

[1] K. Ashton et al., "That internet of things thing," RFID journal, vol. 22, no. 7, pp. 97-114, 2009

[2] F. Colace, M. Lombardi, F. Pascale, D. Santaniello, A. Tucker, and P. Villani, "Mug: A multilevel graph representation for big data interpretation," in 2018 IEEE 20th International Conference on High Performance Computing and Communications; IEEE 16th International Conference on Smart City; IEEE 4th International Conference on Data Science and Systems (HPCC/SmartCity/DSS). IEEE, 2018, pp. 14081413.

[3] Q. Zhang, L. T. Yang, A. Castiglione, Z. Chen, and P. Li, "Secure weighted possibilistic c-means algorithm on cloud for clustering big data," Information Sciences, vol. 479, pp. 515-525, 2019.

[4] G. DAngelo, R. Pilla, J. B. Dean, and S. Rampone, "Toward a soft computing-based correlation between oxygen toxicity seizures and hyperoxic hyperpnea," Soft Computing, vol. 22, no. 7, pp. 2421-2427, 2018.

[5] M. Carratù, M. Ferro, A. Pietrosanto, and P. Sommella, "Wireless sensor network for low-cost air quality measurement," in Journal of Physics: Conference Series, vol. 1065, no. 19. IOP Publishing, 2018, p. 192004.

[6] F. Clarizia, F. Colace, M. Lombardi, F. Pascale, and D. Santaniello, "A multilevel graph approach for road accidents data interpretation," in International Symposium on Cyberspace Safety and Security. Springer, 2018, pp. 303-316.

[7] F. Amato, N. Mazzocca, F. Moscato, and E. Vivenzio, "Multilayer perceptron: An intelligent model for classification and intrusion detection," in 2017 31st International Conference on Advanced Information Networking and Applications Workshops (WAINA). IEEE, 2017, pp. 686-691

[8] G. D'Aniello, M. de Falco, and M. Sergio, "Analysis of the collective perception using granular computing and virtual sensors," in 2018 IEEE Workshop on Environmental, Energy, and Structural Monitoring Systems (EESMS). IEEE, 2018, pp. 1-6.

[9] M. Carratù, M. Ferro, A. Pietrosanto, P. Sommella, and V. Paciello, "A smart wireless sensor network for pm10 measurement," in 2019 IEEE International Symposium on Measurements \& Networking (M\&N). IEEE, 2019, pp. 1-6.

[10] B. Carpentieri, A. Castiglione, A. De Santis, F. Palmieri, and R. Pizzolante, "One-pass lossless data hiding and compression of remote sensing data," Future Generation Computer Systems, vol. 90, pp. 222239, 2019.

[11] F. Colace, M. De Santo, M. Lombardi, F. Pascale, D. Santaniello, and A. Tucker, "A multilevel graph approach for predicting bicycle usage in london area," in Fourth International Congress on Information and Communication Technology. Springer, 2020, pp. 353-362.

[12] F. Colace, M. Lombardi, F. Pascale, and D. Santaniello, "A multi-level approach for forecasting critical events in smart cities," in The 24th International DMS Conference on Visualization and Visual Languages, 2018, pp. 45-46.

[13] F. Colace, M. Lombardi, F. Pascale, and D. Santaniello, "A multilevel graph representation for big data interpretation in real scenarios," in 2018 3rd International Conference on System Reliability and Safety (ICSRS), 2018, pp. 40-47.

[14] S. Kulandaivel, T. Goyal, A. K. Agrawal, and V. Sekar, "Canvas: Fast and inexpensive automotive network mapping," in 28th $\{$ USENIX Security Symposium (\{USENIX\} Security 19), 2019, pp. 389-405. 
[15] C.-W. Lin and A. Sangiovanni-Vincentelli, "Cyber-security for the controller area network (can) communication protocol," in 2012 International Conference on Cyber Security. IEEE, 2012, pp. 1-7.

[16] D. S. Fowler, M. Cheah, S. A. Shaikh, and J. Bryans, "Towards a testbed for automotive cybersecurity," in 2017 IEEE International Conference on Software Testing, Verification and Validation (ICST). IEEE, 2017, pp. 540-541.

[17] T. Hoppe, S. Kiltz, and J. Dittmann, "Security threats to automotive can networkspractical examples and selected short-term countermeasures," Reliability Engineering \& System Safety, vol. 96, no. 1, pp. 11-25, 2011.

[18] K. Koscher, A. Czeskis, F. Roesner, S. Patel, T. Kohno, S. Checkoway, D. McCoy, B. Kantor, D. Anderson, H. Shacham et al., "Experimental security analysis of a modern automobile," in 2010 IEEE Symposium on Security and Privacy. IEEE, 2010, pp. 447-462.

[19] H. Onishi, "Paradigm change of vehicle cyber security," in 2012 4th International Conference on Cyber Conflict (CYCON 2012). IEEE, 2012, pp. 1-11.

[20] J. Reilly, S. Martin, M. Payer, and A. Bayen, "On cybersecurity of freeway control systems: Analysis of coordinated ramp metering attacks," Transportation Research, Part B, 2014.

[21] R. Li, C. Liu, and F. Luo, "A design for automotive can bus monitoring system," in 2008 IEEE Vehicle Power and Propulsion Conference. IEEE, 2008, pp. 1-5.

[22] H. M. Song, H. R. Kim, and H. K. Kim, "Intrusion detection system based on the analysis of time intervals of can messages for in-vehicle network," in 2016 international conference on information networking (ICOIN). IEEE, 2016, pp. 63-68.

[23] M. Casillo, S. Coppola, M. De Santo, F. Pascale, and E. Santonicola, "Embedded intrusion detection system for detecting attacks over canbus," in 2019 4th International Conference on System Reliability and Safety. IEEE, 2019, pp. 136-141.

[24] R. Zalman and A. Mayer, "A secure but still safe and low cost automotive communication technique," in Proceedings of the 51st Annual Design Automation Conference. ACM, 2014, pp. 1-5.

[25] A. Bogdanov, L. R. Knudsen, G. Leander, C. Paar, A. Poschmann, M. J. Robshaw, Y. Seurin, and C. Vikkelsoe, "Present: An ultra-lightweight block cipher," in International workshop on cryptographic hardware and embedded systems. Springer, 2007, pp. 450-466.

[26] R. Beaulieu, S. Treatman-Clark, D. Shors, B. Weeks, J. Smith, and
L. Wingers, "The simon and speck lightweight block ciphers," in 2015 52nd ACM/EDAC/IEEE Design Automation Conference (DAC). IEEE, 2015, pp. 1-6.

[27] "Automotive grade linux," https://www.automotivelinux.org/

[28] R. I. Davis, A. Burns, R. J. Bril, and J. J. Lukkien, "Controller area network (can) schedulability analysis: Refuted, revisited and revised," Real-Time Systems, vol. 35, no. 3, pp. 239-272, 2007.

[29] R. Bosch et al., "Can specification version 2.0," Rober Bousch GmbH, Postfach, vol. 300240, p. 72, 1991.

[30] M. Amiri-Kordestani and H. Bourdoucen, "A survey on embedded open source system software for the internet of things," in Free and Open Source Software Conference, vol. 2017, 2017.

[31] M. J. Cronin, "Ford finds its connection," in Top Down Innovation. Springer, 2014, pp. 13-24.

[32] A. Castiglione, F. Palmieri, F. Colace, M. Lombardi, D. Santaniello, and G. DAniello, "Securing the internet of vehicles through lightweight block ciphers," Pattern Recognition Letters, 2020.

[33] L. Zhou, Q. Wang, X. Sun, P. Kulicki, and A. Castiglione, "Quantum technique for access control in cloud computing ii: Encryption and key distribution," Journal of Network and Computer Applications, vol. 103, pp. 178-184, 2018.

[34] A. Castiglione, A. De Santis, A. Castiglione, and F. Palmieri, "An efficient and transparent one-time authentication protocol with noninteractive key scheduling and update," in 2014 IEEE 28th International Conference on Advanced Information Networking and Applications. IEEE, 2014, pp. 351-358.

[35] A. Castiglione, A. De Santis, B. Masucci, F. Palmieri, and A. Castiglione, "On the relations between security notions in hierarchical key assignment schemes for dynamic structures," in Australasian Conference on Information Security and Privacy. Springer, 2016, pp. 37-54.

[36] A. Castiglione, F. Colace, M. Lombardi, F. Palmieri, and D. Santaniello, "Lightweight ciphers in automotive networks: A preliminary approach," in 2019 4th International Conference on System Reliability and Safety. IEEE, 2019, pp. 142-147.

[37] P. Mundhenk, A. Paverd, A. Mrowca, S. Steinhorst, M. Lukasiewycz, S. A. Fahmy, and S. Chakraborty, "Security in automotive networks: Lightweight authentication and authorization," ACM Transactions on Design Automation of Electronic Systems (TODAES), vol. 22, no. 2, p. $25,2017$. 